\title{
Thermal conductivity across the metal-insulator transition in the single-crystalline hyperkagome antiferromagnet $\mathrm{Na}_{3+x} \mathrm{Ir}_{3} \mathrm{O}_{8}$
}

\author{
B. Fauqué, ${ }^{1,2,{ }^{*}}$ Xiaofeng Xu, ${ }^{1,3}$ A. F. Bangura, ${ }^{1,4}$ E. C. Hunter,${ }^{5}$ A. Yamamoto, ${ }^{4}$ K. Behnia, ${ }^{2}$ A. Carrington, ${ }^{1}$ H. Takagi, ${ }^{4,6}$ \\ N. E. Hussey, ${ }^{1,7}$ and R. S. Perry ${ }^{5,8}$ \\ ${ }^{1}$ H. H. Wills Physics Laboratory, University of Bristol, Tyndall Avenue, Bristol, BS8 1TL, United Kingdom \\ ${ }^{2}$ LPEM (UPMC-CNRS), Ecole Superieure de Physique et de Chimie Industrielles, Rue Vauquelin, 75005 Paris, France \\ ${ }^{3}$ Department of Physics, Hangzhou Normal University, Hangzhou 310036, China \\ ${ }^{4}$ RIKEN (The Institute of Physical and Chemical Research), 2-1 Hirosawa, Wako, Saitama 351-0198, Japan \\ ${ }^{5}$ School of Physics, University of Edinburgh, Mayfield Road, Edinburgh EH9 3JZ, United Kingdom \\ ${ }^{6}$ Department of Physics, University of Tokyo, Hongo, Tokyo, 113-0033, Japan \\ ${ }^{7}$ High Field Magnet Laboratory, Institute for Molecules and Materials, Radboud University Nijmegen, Toernooiveld 7 , \\ 6525 ED Nijmegen, The Netherlands \\ ${ }^{8}$ London Centre for Nanotechnology, 17-19 Gordon Street, University College London, London WC1H OAH, United Kingdom
}

(Received 31 October 2014; revised manuscript received 30 January 2015; published 25 February 2015)

\begin{abstract}
The hyperkagome antiferromagnet $\mathrm{Na}_{4} \mathrm{Ir}_{3} \mathrm{O}_{8}$ represents the first genuine candidate for the realization of a three-dimensional quantum spin liquid. It can also be doped towards a metallic state, thus offering a rare opportunity to explore the nature of the metal-insulator transition in correlated, frustrated magnets. Here, we report thermodynamic and transport measurements in both metallic and weakly insulating single crystals down to $150 \mathrm{mK}$. While in the metallic sample the phonon thermal conductivity $\left(\kappa^{\mathrm{ph}}\right)$ is almost in the boundary scattering regime, in the insulating sample, we find a large reduction $\kappa^{\mathrm{ph}}$ over a very wide temperature range. This result can be ascribed to the scattering of phonons off the gapless magnetic excitations that are seen in the low-temperature specific heat. This works highlights the peculiarity of the metal-insulator transition in $\mathrm{Na}_{3+x} \mathrm{Ir}_{3} \mathrm{O}_{8}$ and demonstrates the importance of the coupling between lattice and spin degrees of freedom in the presence of strong spin-orbit coupling.
\end{abstract}

DOI: 10.1103/PhysRevB.91.075129

PACS number(s): 72.15.Eb, 75.47.Lx

\section{INTRODUCTION}

With their low-energy physical response dominated by geometrical frustration, strong electron correlations and fractionalized elementary excitations, quantum spin liquids (QSLs) occupy a special place in the arena of quantum magnetism. While QSLs have received sustained theoretical attention for many years [1], it is only in the last decade that candidate systems have been discovered [2-4] and only a few of them, like the hyperkagome $\mathrm{Na}_{4} \mathrm{Ir}_{3} \mathrm{O}_{8}$, are based on a three-dimensional (3D) network of corner-sharing triangles. $\mathrm{Na}_{4} \mathrm{Ir}_{3} \mathrm{O}_{8}$ has a Curie-Weiss temperature $\Theta_{\mathrm{CW}}=650 \mathrm{~K}$, yet the localized $S=\frac{1}{2}$ moments on the $\mathrm{Ir}^{4+}$ ions do not order down to temperatures as low as $2 \mathrm{~K}$ [5]. The presence of the $5 d^{5} \mathrm{Ir}$ ions $(Z=77)$ implies that the physics of $\mathrm{Na}_{4} \mathrm{Ir}_{3} \mathrm{O}_{8}$ may also be heavily influenced by strong spin-orbit coupling, prompting a large number of theoretical works exploring various scenarios to describe its magnetic, Mott insulating ground state [6-13].

Specific heat and thermal transport measurements are considered to be insightful, indirect probes of the ground state of candidate QSLs. Specific heat $C$ reveals the presence of lowlying excitations (including those exclusive to spins), while thermal conductivity $\kappa$ indicates if such excitations propagate long enough to be considered delocalized and reveals the strength of coupling between the spin and the lattice degrees of freedom [14]. In some cases, magnetic excitations can contribute significantly to the overall thermal conductivity. For example, in $\left.\mathrm{Et}_{2} \mathrm{Me}_{2} \mathrm{SbPd}_{2} \mathrm{dmit}_{2}\right]_{2}$ (a 2D spin $-\frac{1}{2}$ Heisenberg

\footnotetext{
*benoit.fauque@espci.fr
}

triangular lattice), a residual $T$-linear term was observed in addition to the usual $T^{3}$ phonon contribution [15]. Such a residual $T$-linear contribution to $\kappa$ is not expected in an insulator and thus it has been viewed as evidence for mobile, fermionic gapless excitations of magnetic origin. By contrast, in the 3D classical spin liquid $\mathrm{Tb}_{2} \mathrm{Ti}_{2} \mathrm{O}_{7}$ and the spinel $\mathrm{ZnCr}_{2} \mathrm{O}_{4}$, an extremely low $\kappa$ has been observed [16,17], similar to that found in amorphous solids [14]. There, the marked reduction in the phonon contribution was interpreted as a signature of scattering off strong magnetic fluctuations in the absence of long-range order.

For polycrystalline $\mathrm{Na}_{4} \mathrm{Ir}_{3} \mathrm{O}_{8}$, a $T^{2}$ specific heat contribution of magnetic origin was first reported in Ref. [5], though more recent milli-Kelvin experiments on powdered $\mathrm{Na}_{4} \mathrm{Ir}_{3} \mathrm{O}_{8}$ uncovered an additional $T$-linear term [18]. This latter study also revealed a vanishing $\kappa / T$ as $T \rightarrow 0 \mathrm{~K}$. Measurements on polycrystalline or powdered samples, however, are notoriously susceptible to extrinsic effects, such as impurity contributions in the specific heat or grain boundary scattering of phonons in the low- $T$ thermal conductivity, and it is not clear which of these intriguing observations are intrinsic to $\mathrm{Na}_{4} \mathrm{Ir}_{3} \mathrm{O}_{8}$.

In order to address this issue, we report here an experimental investigation of the specific heat and thermal conductivity of $\mathrm{Na}_{3+x} \mathrm{Ir}_{3} \mathrm{O}_{8}$ single crystals down to millidegree of Kelvin temperatures. The Na deficiency of $\mathrm{Na}_{4} \mathrm{Ir}_{3} \mathrm{O}_{8}$ is found to lead to a doping-induced insulator-metal transition [19] while preserving the chiral hyperkagome lattice of Ir atoms, thereby providing a rare opportunity to explore the Mott insulatormetal transition in a QSL in the presence of a large spin-orbit coupling $[20,21]$. Hereafter, we label our system as $\mathrm{Na}_{3+x} \mathrm{Ir}_{3} \mathrm{O}_{8}$ 
to reflect the fact that the metallic sample $(x=0)$ is more stoichiometric than the insulating sample $(x=0.6)$ studied here. In insulating $\mathrm{Na}_{3+x} \mathrm{Ir}_{3} \mathrm{O}_{8}$, an anomalously small phonon thermal conductivity is observed over a very wide temperature range, indicative of a significant decrease in the phonon mean free path arising from scattering off uncondensed magnetic excitations or nanoscale disorder. In the metallic sample, we observe that phonons are effectively in the boundary scattering regime at low temperature.

\section{CRYSTAL GROWTH AND CHARACTERISATION}

Single crystals of $\mathrm{Na}_{3+x} \mathrm{Ir}_{3} \mathrm{O}_{8}$ were grown using a high temperature flux technique in an oxygen atmosphere. $\mathrm{IrO}_{2}$, $\mathrm{Na}_{2} \mathrm{CO}_{3}$ and $\mathrm{NaCl}$ (flux) were ground together in an agate mortar and pestle in a 1:3:3 ratio. The powders were placed in an alumina crucible with a loose lid and loaded into a tube furnace with baffles. The charge was baked at $1100^{\circ} \mathrm{C}$ for approximately 48 hours at 3 bar gauge oxygen gas pressure. The crystal growth proceeded by flux evaporation with $1 \mathrm{~mm}^{3}$ black crystals growing on the crucible walls. Phase purity and crystallinity were confirmed from powder x-ray diffraction. The sodium concentration of the crystals was determined by electron probe microanalysis (EPMA) with a typical spatial resolution of a few micrometers. Crystals from different batches had notably different properties: crystals quench cooled in oxygen were insulating [red points in Fig. 1(a) and referred to hereafter by the subscripts "ins"], whereas slow cooled $\left(200{ }^{\circ} \mathrm{C} /\right.$ hour $)$ crystals were metallic [blue points on Fig. 1(a) referred to hereafter by the subscripts "met"].

Powder x-ray diffraction on crushed crystals was used to confirm phase purity. The refined structure was identical to previously reported powder samples. The data were refined using GSAS to the structural model provided by Okamoto et al. [5]. The goodness of fit, $\chi^{2}$, was found to be 6.852 and the $R_{\mathrm{wp}}$ was 0.1636 . The lattice parameter was found to be $8.983 \AA$, which is only slightly smaller than the published value of $8.985 \AA$. An important point is that it is very difficult to accurately refine the sodium positions and occupancy because sodium has a far smaller scattering cross-section compared to iridium atoms. We did observe a small unidentifiable impurity



FIG. 1. (Color online) (a) Electrical resistivity $(\rho)$ and (b) magnetic susceptibility $(\chi)$ of $\mathrm{Na}_{3+x} \mathrm{Ir}_{3} \mathrm{O}_{8}$ single crystals as a function of temperature for $x=0.6$ (red open circles) and $x=0.0$ (blue open circles). In this and all subsequent figures, the metal-like $(x=0)$ and insulating $(x=0.6)$ samples are plotted using blue open and red open circles, respectively. phase (possibly $\mathrm{IrO}_{2}$ ) found to make up approximately less than $1 \%$ of the sample as it has been reported in powder samples [22]. Powder X-ray on metallic samples yielded similar results. X-ray Laue backscattering confirms that the samples were single domain.

EPMA on metallic crystals gives the composition to be $\mathrm{Na}_{3} \mathrm{Ir}_{3} \mathrm{O}_{8}$ with the oxygen content fixed at 8 per formula unit. This is compared to $\mathrm{Na}_{3.6} \mathrm{Ir}_{3} \mathrm{O}_{8}$ on the crystals from the insulating batches. We note that EPMA is a compositional analysis tool not without its problems; it is surface sensitive only and less sensitive to light atoms (for example, oxygen). However, the relative error between the insulating and metallic samples should be small. The temperature dependence of the electrical resistivity at both extremes of metallic and insulating behavior is reported in Fig. 1. At room temperature $\rho_{\text {met }}=$ $1.1 \mathrm{~m} \Omega \mathrm{cm}$ and it falls monotonically to $T=25 \mathrm{~K}$, at which point $\rho_{\text {met }}(T)$ develops a small upturn. As $T \rightarrow 0, \rho_{\text {met }}$ levels off at a value of $\approx 600 \mu \Omega \mathrm{cm}$. The high value of $\rho_{\text {met }}$ is consistent with the semimetallic picture for $\mathrm{Na}_{3} \mathrm{Ir}_{3} \mathrm{O}_{8}$ revealed by recent dc [19] and optical conductivity measurements [23]. The effect of the sodium deficiency on $\mathrm{Na}_{3.6} \mathrm{Ir}_{3} \mathrm{O}_{8}$ is to turn the system into a weak insulator characterised by a room temperature resistivity $\rho_{\text {ins }}=40 \mathrm{~m} \Omega \mathrm{cm}$ rising to $130 \mathrm{~m} \Omega \mathrm{cm}$ at low temperature.

The susceptibility for both samples reported on Fig. 1(b), has the same basic behavior as for the powder samples with very similar absolute values. For the insulating sample, the extracted Curie-Weiss temperature is $626 \mathrm{~K}$ with an effective moment of $2.04 \mu_{B} / \mathrm{Ir}$, close to those previously observed $[5,18]$. The susceptibility of the metallic crystals is quite different. It is temperature independent, characteristic of the Pauli paramagnetism. For both samples, we observe an impurity tail at low temperatures $[5,19]$.

\section{THERMAL CONDUCTIVITY MEASUREMENT}

The thermal conductivity measurements were carried out in two stages on two different crystals located at the extremes of the spectrum in $\rho(T)$ behavior, referred to hereafter by the subscripts "met" and "ins." High-temperature $\kappa(T)$ measurements $(10 \mathrm{~K} \leqslant T \leqslant 300 \mathrm{~K})$ were performed using a heat-pipe technique described in detail elsewhere [24], while the low- $T$ measurements were conducted in a dilution refrigerator with a standard one-heater two thermometer setup. The specific heat of single crystals from the same batches (hence showing similar resistivity behavior) was also measured using a relaxation calorimeter described in Ref. [25] and on a Quantum Design PPMS.

As we can see from Fig. 1(b), both thermal conductivities are temperature independent above $T=150 \mathrm{~K}$ with $\kappa_{\text {met }} \approx$ $5 \kappa_{\text {ins }}$. From the difference in the dc conductivities, it is clear that this is not simply due to the additional electronic term $\kappa^{\mathrm{el}}(T)$ in the metallic sample, which, according to the WF ratio $\left[\kappa^{\mathrm{el}} / \sigma T=L_{0}\right.$, where $\left.L_{0}=\left(\pi^{2} / 3\right)\left(k_{B} / e\right)^{2}\right]$, is at least one order of magnitude lower than $\kappa_{\text {met }}(T)$. Thus we can infer that even in the nominally isostructural insulating sample, the phonon thermal conductivity is significantly suppressed over the entire temperature range studied.

Below $100 \mathrm{~K}, \kappa_{\text {met }}$ develops a strong $T$ dependence, passing through a maximum at $T_{\max }=55 \mathrm{~K}$. By contrast, $\kappa_{\text {ins }}$ remains 


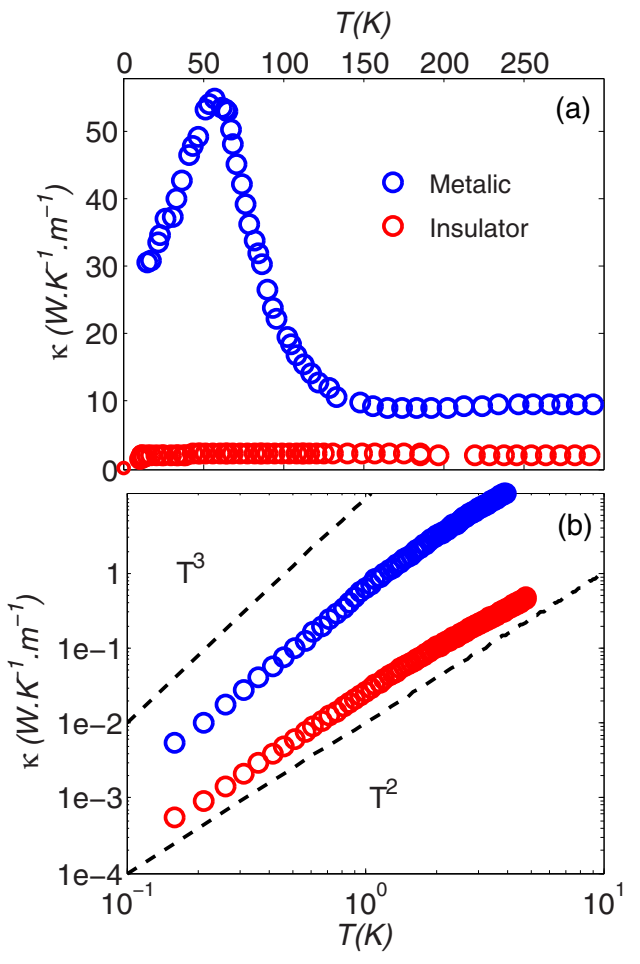

FIG. 2. (Color online) Zero-field thermal conductivity of $\mathrm{Na}_{3+x} \mathrm{Ir}_{3} \mathrm{O}_{8}$ as a function of temperature: (a) between 10 and $300 \mathrm{~K}$ and (b) between $150 \mathrm{mK}$ and $6 \mathrm{~K}$ plotted on a log-log scale.

essentially constant down to temperatures of order $10 \mathrm{~K}$. The peak observed in $\kappa_{\text {met }}$ is the usual behavior expected for the phonon thermal conductivity $\kappa^{\mathrm{ph}}$ of a single crystal, reflecting the balance between the decreasing phonon specific heat and the increasing phonon mean free path [14]. Typically, in doped semiconductors, when the carrier density decreases, the phonon mean free path increases and likewise, the amplitude of the peak increases [26]. In $\mathrm{Na}_{3+x} \mathrm{Ir}_{3} \mathrm{O}_{8}$, we observe the opposite behavior and while insulating $\mathrm{Na}_{3.6} \mathrm{Ir}_{3} \mathrm{O}_{8}$ is expected to be structurally more inhomogeneous (see below), it is clear that we are dealing here with a nontrivial metal-insulator transition.

In order to investigate the origin and evolution of this reduced phonon contribution, we extended our measurements to lower temperature. Figure 2(b) shows a log-log plot of $\kappa_{\text {met }}(T)$ and $\kappa_{\text {ins }}(T)$ between $150 \mathrm{mK}$ and $6 \mathrm{~K}$. Interestingly, we find not only that $\kappa_{\text {met }}$ is significantly enhanced with respect to $\kappa_{\text {ins }}$ (the ratio is now $\frac{\kappa_{\text {met }}}{\kappa_{\text {ins }}} \approx 27$ ) but also that the temperature dependence of $\kappa_{\text {met }}$ and $\kappa_{\text {ins }}$ are fundamentally different. The two distinct power-law behaviors are more clearly seen in Figs. 3(a) and 3(b), where we plot, respectively, $\kappa_{\text {ins, met }} / T$ as a function of $T$ together with the best power law fit to the data below $1 \mathrm{~K}$ : whereas in the metallic case, $\kappa_{\text {met }}$ varies almost as $T^{3}, \kappa_{\text {ins }} \propto T^{2}$. For both samples, we find that a magnetic field affects neither the magnitude nor the $T$ dependence of the thermal conductivity.

A two component fit was used for the metallic sample: $\frac{\kappa_{\text {met }}}{T}=\frac{\kappa^{\mathrm{el}}}{T}+a T^{\alpha}$. The first term corresponds to the electronic part $\kappa^{\mathrm{el}}$, the second term corresponds to the phonon contribution. The phonon contribution to $\kappa_{\text {met }}$ can be expressed


FIG. 3. (Color online) Zero-field thermal conductivity of $\mathrm{Na}_{3+x} \mathrm{Ir}_{3} \mathrm{O}_{8}$ between $150 \mathrm{mK}$ and $6 \mathrm{~K}$ plotted on a log-log scale (a) $\kappa_{\text {ins }} / T$ as a function of temperature for the insulating sample $(0 \mathrm{~T}$, red open circles; $5 \mathrm{~T}$, black closed circles). The black dashed line is a single-component fit to $\frac{\kappa_{\text {ins }}}{T}=b T^{\beta}$ with $b=0.026 \mathrm{~W} \mathrm{~K}^{-(2+\beta)} \mathrm{m}^{-1}$ and $\beta=1.1$. (b) $\kappa_{\text {met }} / T$ as a function of $T^{2}$ for the metallic sample ( $0 \mathrm{~T}$, blue open circles). The black dashed line is a fit to $\frac{\kappa_{\text {met }}}{T}=\frac{\kappa^{\mathrm{el}}}{T}+a T^{\alpha}$, where $\frac{\kappa^{\mathrm{el}}}{T}=\frac{L_{0}}{\rho_{\mathrm{met}}}=4.1 \times 10^{-3} \mathrm{~W} \mathrm{~K}^{-2} \mathrm{~m}^{-1}$, $a=0.61 \mathrm{~W} \mathrm{~K}^{-(2+\alpha)} \mathrm{m}^{-1}$, and $\alpha=1.7$.

through the kinetic formula: $\kappa^{\mathrm{ph}}=\frac{1}{3} C^{\mathrm{ph}}\left\langle v^{\mathrm{ph}}\right\rangle \ell_{0}$, where $\mathrm{C}^{\mathrm{ph}}=\beta^{\mathrm{ph}} T^{3}$ is the phonon specific heat at low temperature, $\left\langle v^{\mathrm{ph}}\right\rangle$ is the average phonon velocity and $\ell_{0}$ is their mean free path. From the specific heat data discussed below, we find $\beta^{\text {ph }}=0.9 \mathrm{~mJ} \mathrm{~K}^{-4} \mathrm{~mol}^{-1}$ and $\left\langle v^{\mathrm{ph}}\right\rangle \approx 2800 \mathrm{~m} \mathrm{~s}^{-1}$, estimated from the Debye temperature $\left(\Theta_{D} \approx 319 \mathrm{~K}\right)$ following Ref. [27]. In the ballistic regime, $\ell_{0} \approx 2 w / \pi$, where $w$ is the average width of the rectangular-shaped crystal $(=0.027 \mathrm{~cm}$ in this case) [28]. The phonon contribution is then expected to be $\kappa^{\mathrm{ph}}=a^{\mathrm{ph}} T^{3}$, where $a=1.4 \mathrm{~W} \mathrm{~K}^{-4} \mathrm{~m}^{-1}$. Assuming the WF law is valid for the electronic contribution $\left(\frac{\kappa^{\mathrm{el}}}{T}=\right.$ $\frac{L_{0}}{\rho_{\text {met }}}=4.1 \times 10^{-3} \mathrm{~W} \mathrm{~K}^{-2} \mathrm{~m}^{-1}$ ), the best fit we find is $a=$ $0.61 \mathrm{~W} \mathrm{~K}^{-(1+\alpha)} \mathrm{m}^{-1}$ and $\alpha_{\mathrm{met}}=1.7$ slightly smaller than the factor two expected in the ballistic regime. The small departure from $T^{3}$ can either occur if the phonons are not wholly in the boundary scattering regime or when the specular reflections are large such as in a dielectric system like $\mathrm{Al}_{2} \mathrm{O}_{3}$ [29]. In our case, since $a \leqslant a^{\mathrm{ph}}$, the deviation from $T^{3}$ is presumably due to the first reason. 


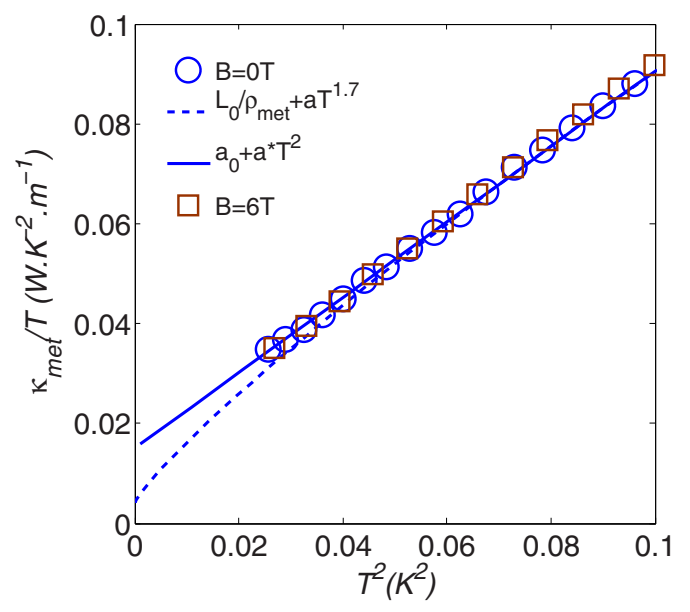

FIG. 4. (Color online) $\kappa_{\mathrm{met}} / T$ as a function of $T^{2}$ for the metallic sample ( $0 \mathrm{~T}$, blue open circles, and $6 \mathrm{~T}$, brown open squares). The blue dash line is a fit to $\frac{\kappa_{\text {met }}}{T}=\frac{L_{0}}{\rho_{\text {met }}}+a T^{\alpha}$, where $\frac{L_{0}}{\rho_{\text {met }}}=$ $4.1 \times 10^{-3} \mathrm{~W} \mathrm{~K}^{-2} \mathrm{~m}^{-1}, a=0.61 \mathrm{~W} \mathrm{~K}^{-(2+\alpha)} \mathrm{m}^{-1}$, and $\alpha=1.7$. The blue line is a fit to $\frac{\kappa_{\text {met }}}{T}=a_{0}+a^{*} T^{2}$, where $a_{0}=1.4 \times 10^{-2} \mathrm{~W} \mathrm{~K}^{-2}$ $\mathrm{m}^{-1}$ and $a^{*}=0.76 \mathrm{~W} \mathrm{~K}^{-4} \mathrm{~m}^{-1}$.

As discussed in the context of high- $T_{c}$ cuprates [30,31], the deviation from a purely $T^{3}$ behavior can significantly affect the estimation of the residual fermionic contribution. In Fig. 4, we report $\frac{\kappa_{\text {met }}}{T}$ as a function of $T^{2}$ from $300 \mathrm{mK}$ down to $150 \mathrm{mK}$. The blue line is the fit previously discussed: $\frac{\kappa_{\text {met }}}{T}=\frac{L_{0}}{\rho_{\text {met }}}+a T^{\alpha}$. The blue dashed line is the textbook fit: $\frac{\kappa_{\text {met }}}{T}=a_{0}+a^{*} T^{2}$, where $a_{0}=1.4 \times 10^{-2} \mathrm{~W} \mathrm{~K}^{-2} \mathrm{~m}^{-1}$ and $a^{*}=0.76 \mathrm{~W} \mathrm{~K}^{-4} \mathrm{~m}^{-1}$. As we can see, the two fits are identical down to $200 \mathrm{mK}$ but the residual intercepts are significantly different. While for the first fit the WF law is verified, for the second fit the residual linear term is three times higher than expected. In the absence of measurements below $150 \mathrm{mK}$, either scenario cannot be ruled out. Yet, such analysis has the merit to quantify the maximum deviation of the WF law. We will come back to this point later.

For the insulating sample, $\kappa(T)$ varies approximately quadratically with temperature from $1 \mathrm{~K}$ down to the lowest temperature investigated. The simplest single-component fit of $\kappa_{\text {ins }}$ is $\frac{\kappa_{\text {ins }}}{T}=b T^{\beta}$ with $b=0.026 \mathrm{~W} \mathrm{~K}^{-\left(2+\alpha_{\text {ins }}\right)} \mathrm{m}^{-1}$ and $\beta=1.1$. This kind of exponent is reminiscent of that seen in the thermal conductivity of glassy materials where it is attributed to resonant phonon scattering off two-level systems associated with tunneling between closely-spaced atomic configurations [32]. The absolute value of $\kappa_{\text {ins }}$ at $T=1 \mathrm{~K}$ is also comparable with that found in amorphous solids such as vitreous silica [32]. For such systems, the $T^{2}$ behavior is associated with a small phonon mean free path that varies as $\ell_{0} \approx 1 / T$ [33]. Following this line of thought, we estimate that the magnitude of the phonon mean free path in the insulating sample is $\ell_{0} \approx 5 \mu \mathrm{m}$ at $T=1 \mathrm{~K}$. This combination of three key observations: the absence of a phonon peak at high temperature, the absence of a $T^{3}$ term in the low- $T$ thermal conductivity and the low value of $\kappa_{\text {ins }}$ all consistently point to an anomalous reduction of the phonon mean free path over the entire temperature range studied.

\section{DISCUSSION}

One possible cause of a reduced $\kappa_{\text {ins }}$ is the disorder associated with the $\mathrm{Na}$ ordering. Although both stoichiometric systems $\left(\mathrm{Na}_{4} \mathrm{Ir}_{3} \mathrm{O}_{8}\right.$ and $\left.\mathrm{Na}_{3} \mathrm{Ir}_{3} \mathrm{O}_{8}\right)$ share the same space group and the same Ir hyperkagome lattice, they differ in the $\mathrm{Na}$ organization. In the simplest picture, the sodium deficiency is attributed to the depopulation of one of the $\mathrm{Na}$ sites of $\mathrm{Na}_{4} \mathrm{Ir}_{3} \mathrm{O}_{8}$, resulting in the presence of vacancies in $\mathrm{Na}_{3.6} \mathrm{Ir}_{3} \mathrm{O}_{8}$. Alternatively, Takayama et al. argued that the Na orderings are different [19], which could lead to the presence of inclusions of $\mathrm{Na}_{3} \mathrm{Ir}_{3} \mathrm{O}_{8}$ in our $\mathrm{Na}_{3.6} \mathrm{Ir}_{3} \mathrm{O}_{8}$ sample. However, we note that the thermodynamic (magnetic susceptibility and specific heat) data for $\mathrm{Na}_{3.6} \mathrm{Ir}_{3} \mathrm{O}_{8}$ are in good agreement with that observed in polycrystalline $\mathrm{Na}_{4} \mathrm{Ir}_{3} \mathrm{O}_{8}$ [5], and quite distinct from what is found in the metallic sample. This suggests that the insulating behavior of our crystals is intrinsic and is not simply due to poor sample quality. The distinction between both types of defects is not easy to make due to the poor sensitivity of $x$ rays to Na sites in the Ir matrix. Both defects (vacancies or inclusions) can act as scattering centers for the phonons and can thus give rise to a reduced $\kappa_{\text {ins }}$ when the phonon wave length is of the same order of magnitude as the characteristic length scale of the defects. However, neither defect can explain on its own the reduced $\kappa_{\text {ins }}$ over the full temperature range. It is interesting to note that in the case of gadolinium gallium garnet $\mathrm{Gd}_{3} \mathrm{GaO}_{5} \mathrm{O}_{12}$ (a $3 \mathrm{D}$ hyperkagome lattice), oxygen vacancies do not change the amplitude nor the temperature dependence of the thermal conductivity [34] illustrating the fact that phonons at low temperature are only weakly affected by local defects. At low temperature, the phonon mean free path is about $\approx 1 \mu \mathrm{m}$, a length scale at which the Na stoichiometry is homogenous (as shown by EPMA) and much larger than the distance between $\mathrm{Na}$ vacancies. Beyond the effect of disorder, additional scattering processes must be invoked to explain the poor thermal conductivity of our weakly insulating $\mathrm{Na}_{3+x} \mathrm{Ir}_{3} \mathrm{O}_{8}$ crystal.

The findings reported here are highly reminiscent of the phonon-glass-like behavior recently reported in $\mathrm{Tb}_{2} \mathrm{Ti}_{2} \mathrm{O}_{7}$ $[16,17]$. There, it was argued that the small energy separation between the crystal field ground state and the first excited state is the essential ingredient that leads to a strong reduction of $\ell_{0}$. In particular, the complicated field dependence of the specific heat and thermal conductivity observed in $\mathrm{Tb}_{2} \mathrm{Ti}_{2} \mathrm{O}_{7}$ has been discussed in the context of spin-splitting of the crystal field levels [16]. In Fig. 5(a), we plot the temperature dependence of the specific heat of our insulating sample for three magnetic field strengths, $B=0,6$, and $12 \mathrm{~T}$. In marked contrast to what is seen in $\mathrm{Tb}_{2} \mathrm{Ti}_{2} \mathrm{O}_{7}$, a magnetic field appears to have no effect on the absolute value of either the specific heat (in good agreement with an earlier powder measurement [5]) or the thermal conductivity. In the light of this finding, it appears that the crystal field plays no role in our weakly insulating $\mathrm{Na}_{3+x} \mathrm{Ir}_{3} \mathrm{O}_{8}$ crystal.

A comparison of the specific heat of the metallic and insulating samples provides a possible clue for the reduction of $\kappa_{\text {ins. }}$. As reported in Figs. 5(b) and 5(c), $C_{\text {met }}$ and $C_{\text {ins }}$ display markedly different behavior at low temperatures. Below $5 \mathrm{~K}$, in good agreement with [19], $C_{\mathrm{met}}(T)$ is well captured by a two component fit $\gamma_{\mathrm{met}} T+\beta_{\mathrm{met}} T^{3}$, where 

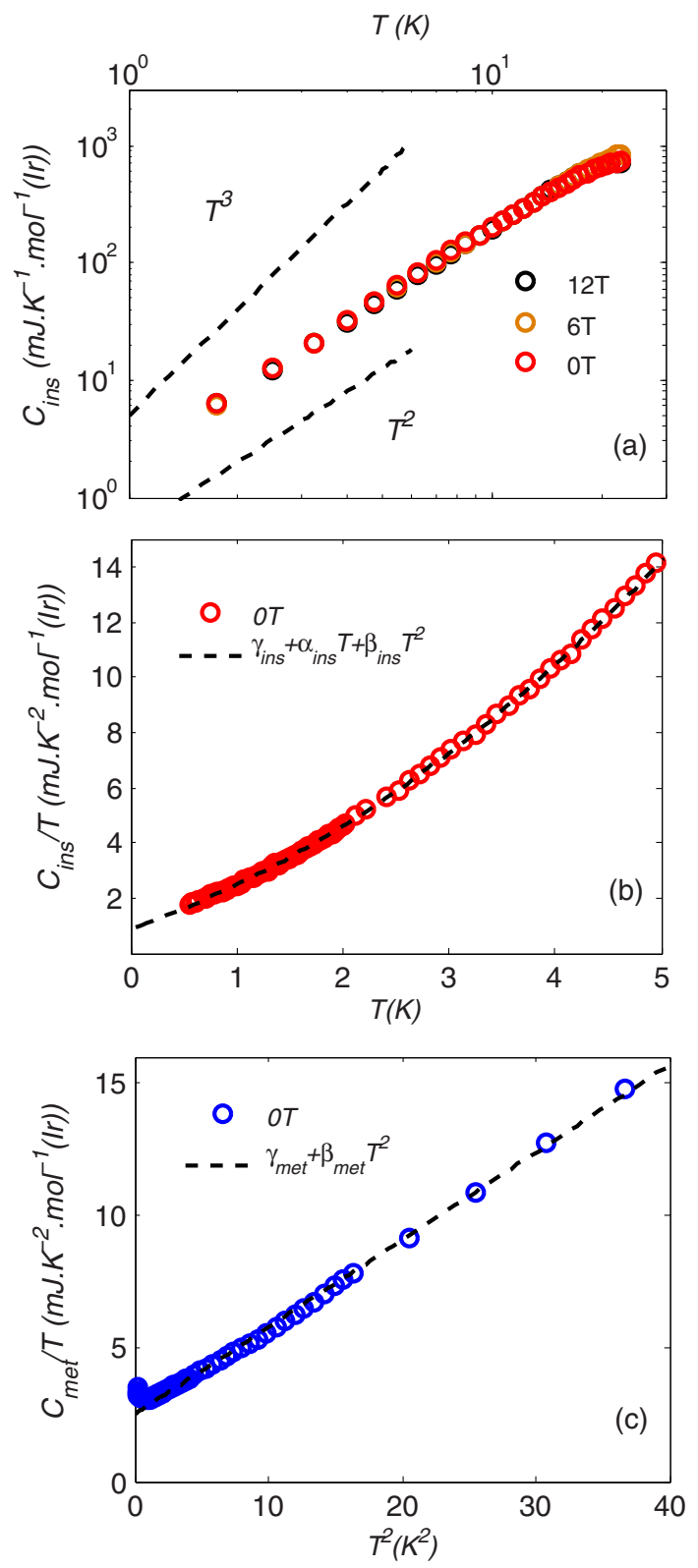

FIG. 5. (Color online) (a) Temperature dependence of the specific heat $C_{\text {ins }}(T)$ of insulating $\mathrm{Na}_{3+x} \mathrm{Ir}_{3} \mathrm{O}_{8}$ in magnetic fields of 0 (red), 6 (orange), and $12 \mathrm{~T}$ (black open circles) plotted on a log-log scale. (b) Low- $T$ magnetic specific heat of the insulating sample $\left(C_{\text {ins }}\right)$ divided by $T$ as function of $T$. The black line is a fit of $C_{\mathrm{ins}}(T)=$ $\gamma_{\text {ins }} T+\alpha_{\text {ins }} T^{2}+\beta_{\text {ins }} T^{3}$ from 0.5 to $5 \mathrm{~K}$. The values of $\gamma_{\text {ins }}, \alpha_{\text {ins }}$ and $\beta_{\text {ins }}$ are given in the text. (c) $C_{\text {met }} / T$ as a function of $T^{2}$. The black dotted line is a fit to $C_{\text {met }}(T)=\gamma_{\text {met }} T+\alpha_{\text {met }} T^{3}$ where $\gamma_{\text {met }}$ and $\alpha_{\text {met }}$ are given in the text.

$\gamma_{\text {met }}=2.4 \mathrm{~mJ} \mathrm{~K}^{-2} \mathrm{~mol}^{-1}$ (Ir) and $\beta_{\text {met }}=0.32 \mathrm{~mJ} \mathrm{~K}^{-4} \mathrm{~mol}^{-1}$ (Ir). We note that below $1 \mathrm{~K}$ a small upturn is observed, presumably due to disorder [35]. For the insulating sample, as highlighted in Fig. 5(a), the full specific heat is dominated by a $T^{2}$ term above $1 \mathrm{~K}$ with an extra contribution below $1 \mathrm{~K}$. Therefore, in order to fit across the whole temperature region, a third component has to be added to fit the $C_{\text {ins }}=\gamma_{\text {ins }} T+\alpha_{\text {ins }} T^{2}+\beta_{\text {ins }} T^{3}$, where $\gamma_{\text {ins }}=1.2 \mathrm{~mJ} \mathrm{~K}^{-2}$ $\mathrm{mol}^{-1}$ (Ir), $\quad \alpha_{\text {ins }}=1.0 \mathrm{~mJ} \mathrm{~K}^{-3} \mathrm{~mol}^{-1}(\mathrm{Ir}), \quad$ and $\beta_{\text {ins }}=$
$0.31 \mathrm{~mJ} \mathrm{~K}^{-3} \mathrm{~mol}^{-1}$ (Ir). Note that the $T^{3}$ terms are almost identical, suggesting that there is no additional $T^{3}$ term in the insulating sample coming from the presence of two-levelsystems [36]. Intriguingly, the $\gamma$ term does not change significantly across the metal-insulator transition $\left(\gamma_{\text {met }} \approx 2 \gamma_{\text {ins }}\right)$. It is therefore tempting to ascribe the additional $T^{2}$ term in the specific heat to the same excitations that are responsible for the strong reduction of the phonon mean free path in insulating $\mathrm{Na}_{3.6} \mathrm{Ir}_{3} \mathrm{O}_{8}$. Indeed, this enigmatic $T^{2}$ term is observed in other kagome lattices $[37,38]$. It is also noted that the sample with the highest magnetic specific heat (above $1 \mathrm{~K}$ ) has the smallest thermal conductivity. This unexpected finding reveals the complex interplay between the magnetic and phononic degrees of freedom in a system with a large spin-orbit coupling.

One outstanding question of this work is the contribution of the magnetic excitations to the thermal conductivity. Recent investigations on powdered $\mathrm{Na}_{4} \mathrm{Ir}_{3} \mathrm{O}_{8}$ find an upper limit to the residual linear contribution of $\kappa_{\text {ins }}(0) / T=$ $6.3 \times 10^{-2} \mathrm{~mW} \mathrm{~K}^{-2} \mathrm{~m}^{-1}$ [18]. Outside of the ballistic regime, any extrapolation of the phonon contribution, and by inference, of the magnetic contribution, cannot be done reliably. Nevertheless, it is clear that the magnetic contribution to $\kappa_{\text {ins }}$ is extremely small, at least three orders of magnitude smaller than that found in $\mathrm{Et}_{2} \mathrm{Me}_{2} \mathrm{SbPd}\left[\mathrm{dmit}_{2}\right]_{2}$ [15]. In the metallic sample, the ballistic regime is almost attained. A finite linear term can be resolved: it is at maximum, three times larger than what is predicted by the WF law. This deviation could have various origins. It could be associated with the proximity to the metal-insulator transition, as seen for example in underdoped high- $T_{c}$ cuprates [39] where the deviation has been attributed to intrinsic disorder associated with carrier doping. It could also be associated with the compensated nature of the electronic structure of $\mathrm{Na}_{3} \mathrm{Ir}_{3} \mathrm{O}_{8}$ [19]. Here, a third term has to be added to $\kappa$ in addition to the phonic and electronic contributions. This third term, the bipolar thermodiffusion, is the result of the heat transport of electron-hole pairs [40]. In semimetals such as bismuth, this term can be as high as one quarter of the electronic contribution at high temperature [41]. This contribution generally collapses at low temperature where the WF law is obeyed [42,43]. However, the striking similarity of the $\gamma$ terms in both the metallic and insulating samples may indicate another origin for this deviation. While in the insulating sample, the residual specific heat can be attributed to gapless magnetic excitations [18], an assignment of $\gamma_{\text {met }}$ purely to the conduction electrons [19] is not as clear-cut. Indeed, close to the metal-insulator transition, the distinction between each degree of freedom becomes much less straightforward. Thus it is feasible that an additional residual term in $\kappa_{\text {met }}$ (i.e., above the WF law estimate) could be due to a residue of fermionic magnetic excitations. Roughly speaking, if we assume that $\gamma_{\text {met }}$ is due entirely to magnetic excitations (and assuming that the sound velocity of these excitations $\mathrm{v}_{\text {mag }} \approx 10^{5} \mathrm{~m} \mathrm{~s}^{-1}$ ), then their maximum mean free path for the magnetic excitation is estimated to be of the order of $10 \mathrm{~nm}$ (i.e., $\sim 10$ unit cells), higher than in the insulating sample, but still two orders of magnitude lower than what has been reported for $\mathrm{Et}_{2} \mathrm{Me}_{2} \mathrm{SbPd}\left[\mathrm{dmit}_{2}\right]_{2}$. It would appear then that while the magnetic specific heat is large, the magnetic excitations in $\mathrm{Na}_{3+x} \mathrm{Ir}_{3} \mathrm{O}_{8}$ are either localised (on the insulating side) or have a very short mean free-path (on the metallic side), resulting in 
a poor thermal conductivity. This picture is in deed consistent with a recent $\mu \mathrm{SR}$ study where the spin correlations have been found short-range [44].

\section{CONCLUSION}

In conclusion, by comparing the behavior of two singlecrystalline samples located on either side of the (albeit weak) metal-insulator boundary, we have uncovered signatures of a significant damping of the phonon mean free path in the frustrated hyperkagome antiferromagnet $\mathrm{Na}_{3+x} \mathrm{Ir}_{3} \mathrm{O}_{8}$. This damping may arise from strong coupling of the lattice degrees of freedom with unconventional low-lying spin excitations, possibly mediated through the enhanced spin-orbit coupling of the $\mathrm{Ir}^{4+}$ ions. This work has also highlighted the very distinct behavior seen in the specific heat and thermal conductivity of different candidate quantum spin liquids, presenting a significant challenge to those seeking a unified theoretical description. Looking forward, it would be interesting to explore at lower temperature the possible deviation of the WF law across the metal-insulator transition and to investigate whether such strong spin-orbit coupling also influences the low- $T$ thermodynamic and transport properties of other insulating iridates, such as the $j_{\text {eff }}=\frac{1}{2}$ Mott insulator $\mathrm{Na}_{2} \mathrm{IrO}_{3}$.

\section{ACKNOWLEDGMENTS}

B.F. thank useful discussions with Julien Robert and Michel Gingras. This work was supported by EPSRC (EP/G031460) and by a Royal Society Wolfson Research Merit Award (N.E.H.). B.F. received financial support through a Newton International Fellow award and the Emergence-UPMC program.

\section{APPENDIX A: ANNEALING STUDY ON AS GROWN SAMPLE}

We performed an annealing study on an as-grown, insulating single crystal of $\mathrm{Na}_{3.6} \mathrm{Ir}_{3} \mathrm{O}_{8}$. After each treatment (see Fig. 6 description for details), the magnitude of the resistivity and its temperature gradient monotonously decreased until a quasimetallic dependence was observed. Powder x-ray analysis on the crystal after the study showed no evidence of decomposition. A mass loss study was also undertaken in a thermogravimetric analyzer using identical conditions to the resistivity study. We measured a decrease in mass of $\approx 2 \%$ total over the entire study. An excess of oxygen can also occurred during the heat treatment. Both effects will hole doped the conductions bands resulting in a metallic state. If we assume only sodium is lost, we can estimate a change in sodium concentration per formula unit of 0.7 over the entire experiment, in reasonable agreement with compositional analyses of as-grown crystals suggesting that the change in oxygen stoichiometry is indeed rather limited.

\section{APPENDIX B: COMPARISON BETWEEN POWDER AND SINGLE CRYSTAL MEASUREMENTS}

We reported on the left panel of Fig. 7(a) comparison of the magnetic specific heat from our single crystal $\mathrm{Na}_{3.6} \mathrm{Ir}_{3} \mathrm{O}_{8}$ and for the powder $\mathrm{Na}_{4} \mathrm{Ir}_{3} \mathrm{O}_{8}$ [5]. No difference is observed.



FIG. 6. Annealing study on insulating crystals of $\mathrm{Na}_{3+x} \mathrm{Ir}_{3} \mathrm{O}_{8}$. The crystal was initially insulating (top curve). It was repeatedly heattreated between $300{ }^{\circ} \mathrm{C}$ and $500{ }^{\circ} \mathrm{C}$ for 12 hours in flowing oxygen and measured between each treatment. Electrical contacts $(50-\mu \mathrm{m}$ gold wire attached with Dupont 4929 silver epoxy) were kept on the crystal to ensure that the relative error in the absolute magnitude of the resistivity was minimized between measurements.

As well the magnetic susceptibility has the same behavior as the powder $\mathrm{Na}_{4} \mathrm{Ir}_{3} \mathrm{O}_{8}$. The extracted Curie-Weiss temperature from Fig. 7(b) is $626 \mathrm{~K}$ with an effective moment of $2.04 \mu_{B} / \mathrm{Ir}$, close to those previously observed [5,18].

\section{APPENDIX C: PHONON CONTRIBUTION IN THE SPECIFIC HEAT}

Due to the large magnetic contribution in the specific heat for magnetic systems, the phonon contribution is determined independently via a measurement of the specific heat in isostructural, nonmagnetic system. In the case of $\mathrm{Na}_{4} \mathrm{Ir}_{3} \mathrm{O}_{8}$, the phonon contribution has been estimated so far by the measurement in $\mathrm{Na}_{4} \mathrm{Sn}_{3} \mathrm{O}_{8}[5,18]$. Below $30 \mathrm{~K}$, the specific heat of $\mathrm{Na}_{4} \mathrm{Sn}_{3} \mathrm{O}_{8}$ is well described by $C_{\text {pho }}=\beta_{\text {pho }} T^{3}$, where $\beta_{\text {pho }}=0.45 \mathrm{~mJ} \mathrm{~K}^{-4} \mathrm{~mol}^{-1}$. As reported in the manuscript, below $10 \mathrm{~K}$, we found, within the error of the fit, the same $T^{3}$ contribution in the specific heat for both metallic and insulator sample equal to $0.9 \mathrm{~mJ} \mathrm{~K}^{-4} \mathrm{~mol}^{-1}$. According to Takayama et al. [19] even if $\mathrm{Na}_{4} \mathrm{Ir}_{3} \mathrm{O} 8$ and $\mathrm{Na}_{3} \mathrm{Ir}_{3} \mathrm{O} 8$ belong to the same group symmetry ( $P 4132$ or $P 4332$ ) and share in common the same Ir-hyperkagome lattice, the two systems have a distinct
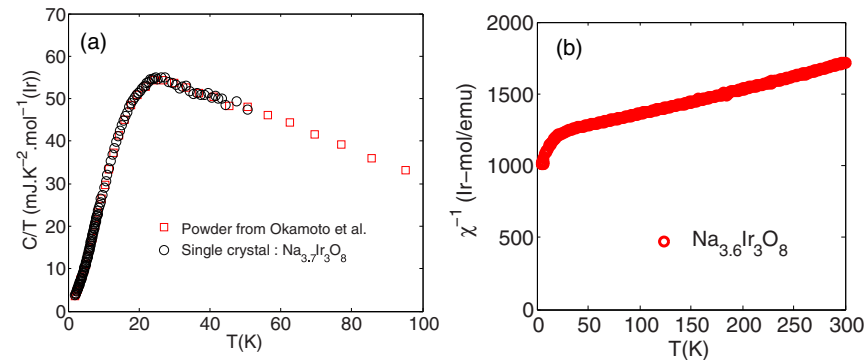

FIG. 7. (Color online) (a) Comparison of the magnetic specific heat divided by temperature as function of the temperature from Okamoto et al. (red points) [5] and measured on the single-crystal $\mathrm{Na}_{3.6} \mathrm{Ir}_{3} \mathrm{O}_{8}$ [black points]. (b) $\chi^{-1}$ as a function of the temperature for the single-crystal $\mathrm{Na}_{3.6} \operatorname{Ir}_{3} \mathrm{O}_{8}$ 
structure. However, the $T^{3}$ term as observed in the specific heat is insensitive to the subtle difference between the two structures. While in the main text, we attribute the $T^{3}$ term to a purely phononic origin, the comparison with $\mathrm{Na}_{4} \mathrm{Sn}_{3} \mathrm{O}_{8}$ suggests another interpretation. The factor two of difference could be the result of the contribution of magnetic excitations to the specific heat in both metallic and insulating samples, in addition to the phonon contribution. While this hypothesis cannot be exclude in the insulating sample, it seems, at first sight, unlikely in the case of the metallic sample, which displays a Pauli magnetic susceptibility (see Fig. 1). In other words, the peak observed at $30 \mathrm{~K}$ in the magnetic specific heat (and which is independent of the doping [22]) could be simply attributed to the difference in the phonon specific heat between $\mathrm{Na}_{4} \mathrm{Ir}_{3} \mathrm{O}_{8}$ and $\mathrm{Na}_{4} \mathrm{Sn}_{3} \mathrm{O}_{8}$. Finally, we note that if we take the phonon contribution deduced from $\mathrm{Na}_{4} \mathrm{Sn}_{3} \mathrm{O}_{8}\left(\beta_{\text {pho }}=\right.$ $0.45 \mathrm{~mJ} \mathrm{~K}^{-4} \mathrm{~mol}^{-1}$ ), our conclusion regarding the ballistic regime in the metallic sample is not affected at all. In fact, using this value, the mean free path of the phonon is even closer to $w$.
[1] P. W. Anderson, Mater. Res. Bull. 8, 153 (1973).

[2] P. A. Lee, Science 321, 1306 (2008).

[3] L. Balents, Nature (London) 464, 199 (2010).

[4] M. J. P. Gingras and P. A. McClarty, Rep. Prog. Phys. 77, 056501 (2014)

[5] Y. Okamoto, M. Nohara, H. Aruga-Katori, and H. Takagi, Phys. Rev. Lett. 99, 137207 (2007).

[6] J. M. Hopkinson, S. V. Isakov, H.-Y. Kee, and Y. B. Kim, Phys. Rev. Lett. 99, 037201 (2007).

[7] M. J. Lawler, H. Y. Kee, Y. B. Kim, and A. Vishwanath, Phys. Rev. Lett. 100, 227201 (2008).

[8] Y. Zhou, P. A. Lee, T.-K. Ng, and F.-C. Zhang, Phys. Rev. Lett. 101, 197201 (2008)

[9] M. J. Lawler, A. Paramekanti, Y. B. Kim, and L. Balents, Phys. Rev. Lett. 101, 197202 (2008).

[10] G. Chen and L. Balents, Phys. Rev. B 78, 094403 (2008)

[11] M. R. Norman and T. Micklitz, Phys. Rev. B 81, 024428 (2010).

[12] E. J. Bergholtz, A. M. Lauchli, and R. Moessner, Phys. Rev. Lett. 105, 237202 (2010).

[13] G. Chen and Y. B. Kim, Phys. Rev. B 87, 165120 (2013).

[14] R. Berman, Thermal Conduction in Solids (Clarendon Press, Oxford, 1976).

[15] M. Yamashita, N. Nakata, Y. Senshu, M. Nagata, H. M. Yamamoto, R. Kato, T. Shibauchi, and Y. Matsuda, Science 328, 1246 (2010).

[16] Q. J. Li, Z. Y. Zhao, C. Fan, F. B. Zhang, H. D. Zhou, X. Zhao, and X. F. Sun, Phys. Rev. B 87, 214408 (2013).

[17] M. Tachabina, Solid State Commun. 174, 16 (2013).

[18] Y. Singh, Y. Tokiwa, J. Dong, and P. Gegenwart, Phys. Rev. B 88, 220413(R) (2013).

[19] T. Takayama et al., Sci. Rep. 4, 6818 (2014).

[20] D. Podolsky, A. Paramekanti, Y. B. Kim, and T. Senthil, Phys. Rev. Lett. 102, 186401 (2009).

[21] D. Podolsky and Y. B. Kim, Phys. Rev. B 83, 054401 (2011).

[22] A. Balodhi, A. Thamizhavel and Yogesh Singh, arXiv:1412.0455v1.

[23] D. Pröpper, A. N. Yaresko, T. I. Larkin, T. N. Stanislavchuk, A. A. Sirenko, T. Takayama, A. Matsumoto, H. Takagi, B. Keimer, and A. V. Boris, Phys. Rev. Lett. 112, 087401 (2014).
[24] N. Wakeham, A. F. Bangura, X. Xu, M. Greenblatt, and N. E. Hussey, Nat. Commun. 2, 396 (2011).

[25] O. J. Taylor, A. Carrington, and J. A. Schlueter, Phys. Rev. Lett. 99, 057001 (2007).

[26] J. A. Carruthers, T. H. Geballe, H. M. Rosenberg, and J. M. Ziman, Proc. R. Soc. A 238, 502 (1957)

[27] Z. Y. Zhao, X. M. Wang, C. Fan, W. Tao, X. G. Liu, W. P. Ke, F. B. Zhang, X. Zhao, and X. F. Sun, Phys. Rev. B 83, 014414 (2011).

[28] N. E. Hussey, S. Nakamae, K. Behnia, H. Takagi, C. Urano, S. Adachi, and S. Tajima, Phys. Rev. Lett. 85, 4140 (2000).

[29] R. O. Pohl and B. Stritzker, Phys. Rev. B 25, 3608 (1982).

[30] S. Y. Li, J.-B. Bonnemaison, A. Payeur, P. Fournier, C. H. Wang, X. H. Chen, and Louis Taillefer, Phys. Rev. B 77, 134501 (2008).

[31] S. Y. Li, J.-B. Bonnemaison, A. Payeur, P. Fournier, C. H. Wang, X. H. Chen, and Louis Taillefer, Phys. Rev. B 79, 176502 (2009); X. F. Sun and Yoichi Ando, ibid. 79, 176501 (2009)

[32] R. C. Zeller and R. O. Pohl, Phys. Rev. B 4, 2029 (1971).

[33] P. W. Anderson, B. I. Halperin, and C. M. Varma, Philos. Mag. 25, 1 (1972).

[34] B. Daudin, R. Lagnier, and B. Salce, J. Magn. Magn. Mater. 27, 315 (1982).

[35] G. A. Thomas, Y. Ootuka, S. Kobayashi, and W. Sasaki, Phys. Rev. B 24, 4886 (1981).

[36] J. J. De Yoreo, W. Knaak, M. Meissner, and R. O. Pohl, Phys. Rev. B 34, 8828 (1986).

[37] S. Nakatsuji et al., Science 309, 1697 (2005).

[38] T.-H. Han, R. Chisnell, C. J. Bonnoit, D. E. Freedman, V. S. Zapf, N. Harrison, D. G. Nocera, Y. Takano, and Y. S. Lee, arXiv:1402.2693.

[39] Cyril Proust, Kamran Behnia, Romain Bel, Duncan Maude, and S. I. Vedeneev, Phys. Rev. B 72, 214511 (2005).

[40] C. F. Gallo, R. C. Miller, P. H. Sutter, and A. W. Ure, J. Appl. Phys. 33, 3144 (1962)

[41] C. F. Gallo, B. S. Chandrasekhar, and P. H. Sutter, J. Appl. Phys. 34, 144 (1963)

[42] C. K. Chau and S. Y. Lu, J. Low Temp. Phys. 15, 447 (1974).

[43] W. P. Pratt Jr. and C. Uher, Phys. Lett. A 68, 74 (1978).

[44] R. Dally et al., arXiv:1410.7072. 